\title{
The pitfalls of dose titration as used in electroconvulsive therapy
}

\author{
P. O. Byme, R. G. Farquharson and I. S. Schofield
}

\begin{abstract}
Dose throtion is the now colloquialism in ECT clrcles. The techniqus, as generolly applied, casumes that current and the time for which it is paseed, are equally important in the depolatication of nerve cella. The units uesed to quantioy the mecaurement, nomoty the minic coulomb (mC) reflect this. We think this bs misibading and rugeret it ought not to be uned as a porameler to describe a trectiment recims. If is the cim of this communication to explain, in general terms, the physical poramoters that control the current flowing in the potient's hoad, to rugoest a more appropilate mothod to describe the treatment odminietered and outiline the requirements of a good ECT mochine.
\end{abstract}

\section{The patient's head}

No two people are identical. The structure of the head has a number of physical variables which will affect the flow path of current, for example skull thickness, bone density and porosity. In addition, factors such as room temperature, anxiety, age and drug therapy also modify the patient's expected physiological response to current stimulation. In electrical terms, the patient presents an impedance to the current flow but Offner (1942) showed that for practical purposes the patient circuit could be purely resistive.

Living bone, compared to soft tissue, is a very good insulator. Typically, tissue and brain have a specific resistance of $220 \mathrm{ohm}$ metre whereas bone presents a specific resistance of about $18000 \mathrm{ohm}$ metre (Weaver et al, 1976). Hence the skull effectively insulates or shields the brain from the majority of the stimulating current. The scalp and its associated blood supply acts as a shunt or bypass for the current delivered by the ECT machine and for a given patient this will vary by some small but unpredictable amount from day to day. The theoretical model used by Weaver et al. suggests that as much as $64 \%$ of the applied current is shunted by the scalp for unilateral placement of the electrodes.

It is possible to condition the skin to conduct electricity by exploiting the mechanisms of iontophoresis and transdermal electroporation. This is done by utilising pulses milliseconds long with rise times of tens of microseconds and voltages in the order of one hundred volts. Providing the electrode sites are cleaned and the dampened electrodes are held against the head with a firm constant pressure throughout the treatment the patient circuit will always present an essentially constant resistive load to the ECT machine of about 200 ohms. Hence there is no need whatsoever to measure electrode-skin contact impedance.

\section{The current threshold}

To be clinically effective ECT must induce a seizure in the patient. This is achieved by passing a train of current pulses through the patient's head of the correct magnitude for a period of time necessary to initiate a seizure. Clinical observations have shown that providing the current is above some threshold, which has broad limits, the stimulation time need only be a few seconds. If, however, the magnitude of the current is below the threshold needed to initiate a seizure focus, increasing the stimulation time will serve no clinically useful purpose. Each patient is unique from an electrical point of view and at their first visit to the ECT clinic their individual current threshold must be established. This is best done by fixing the following stimulation parameters to the values shown below, for example.
(a) Stimulation time
(b) Pulse width
3 seconds
(c) Frequency
1 millisecond
$100 \mathrm{~Hz}$

With the aid of a controllable constant current stimulator the stimulating current is set to a suitable value, the following figures are offered as a guide.

$\begin{array}{ll}\text { (a) A young person } & 350 \mathrm{~mA} \\ \text { (b) A middle-aged person } & 550 \mathrm{~mA} \\ \text { (c) An old person } & 750 \mathrm{~mA}\end{array}$ up to say $1000 \mathrm{~mA}$ 
Give the stimulation and observe the results. If the fit is prolonged reduce the current by 50 or $100 \mathrm{~mA}$ at the next clinic visit. If the patient does not fit then increase the current by 50 or $100 \mathrm{~mA}$ and try again. In this situation it is recommended that the patient should only be stimulated a maximum of three times at any one visit. The aim of this exercise is to produce a well modified fit by optimising the current for the individual patient. During the course of the treatment it may be necessary to increase the stimulation (Sackeim et al, 1991) by a small amount at each visit, this relates to an increase in current of say 10 to $35 \mathrm{~mA}$. However, in all cases the observed quality of the fit must influence the current setting used and this value should be recorded in the patient's notes.

\section{The measurement units}

Sackeim et al (1987) used a purpose-built constant current ECT machine designed to give a fixed current of $800 \mathrm{~mA}$. By multiplying this current by the stimulus time they obtained the electrical unit of charge (current $x$ seconds $\times$ pulsewidth $\times$ frequency) called the Coulomb (C) or milliCoulomb (mC). In their subsequent work they correctly used this as a treatment index to describe their treatment regime and results. However, this technique cannot be untversally applied because ECT machines differ widely. Some are constant voltage while others purport to be constant current but their outputs are not fixed by a recognised standard. In addition, a stimulus of $800 \mathrm{~mA}$ with a pulse width of $2 \mathrm{~ms}$ at a frequency of $100 \mathrm{~Hz}$ applied for 2 seconds $(160 \mathrm{mC})$ produces a totally different physiological response to $320 \mathrm{~mA}$ applied for 5 seconds $(160 \mathrm{mC})$ in a patient whose current threshold is $600 \mathrm{~mA}$. Clearly this unit is totally unsuitable for prescribing the electrical dose to a patient. Current is the dominating stimulus parameter, so by fixing the stimulus time. frequency, pulse width and its associated parameters to some nationally agreed values, like those shown above, the magnitude of the current used would then describe accurately the treatment criteria for a given patient.

\section{The ECT machine}

Simple to use constant current machines are preferred for many reasons but principally because they allow the clinician to describe, with a degree of accuracy, what the patient received and relate this to the clinical result.
Also, it enables clinicians to compare results nationally and internationally on a sound scientific footing.

Electrical parameters such as frequency and pulse width for all intended purposes could be fixed. The rise and fall time for each pulse should be better than 25 microseconds for reasons outlined above. The pulse width should be measured at the $10 \%$ points to an accuracy of $\pm 10 \%$, a similar criterion of $\pm 10 \%$ should apply to the frequency. The output pulse should be bi-phasic as this reduces the likelihood of ions from the electrodes being driven into the skin during stimulation.

It is a very costly exercise to integrate EEG recording facilities into an ECT machine and the additional time spent setting up the patient in a busy clinic for recording would be hard tojustify for the very rare occurrence of status epilepticus. While EEG activity is the ultimate arbitrator, it is almost impossible, from an EEG trace, to judge the seizure end point with any degree of confidence. EMG and ECG activity (Abrams, 1992) can also be used to indicate that the patient is fitting. With no clear national recommendations from governing bodies the value of recording these parameters from the patient must be questioned.

A complete specification on safety and construction requirements for ECT machines can be found in BS 5724:1989 Section 2.14. Purchasers of such equipment should satisfy themselves that these standards are met or that the manufacturer has clearly specified areas of non-compliance in the MLQ forms.

\section{References}

ABRAMs, R (1992) Electroconurulsive Therapy. New York: Oxford Untversity Press.

OFFNER, F. (1942) Electrical properties of tissues in shock therapy. Proceedings of the Society of Experimental Biology and Medictne, 49, 571-574.

SACKend, H. A., Devanand, D. P. \& PRUdiC, J. (1991) Stimulus intensity, seizure threshold, and setzure duration. Psychiatic Clinics of North America, 14, 803-843.

- Decina, P., Prohovnik, I. \& MuLtz, S. (1987) Selzure threshold in electroconvulstve therapy. Archives of General Psychiatry. 44, 355-360.

Weaver, L., Wunams, R., \& RUSh, S. (1976) Current density In bilateral and unilateral ECT. Biological Psychiatry. 11. 303-312.

P. O. Byrne, Head of Clinical Instrumentation, Regional Medical Physics Department, R. G. Farquharson, Consultant Psychiatrist, and I. S. Schofield, Consultant Neurophysiologist, Newcastle General Hospital, Neucastle Upon Tyne NE4 6BE 